\title{
Ericaceae endémicas del Perú
}

\author{
Blanca León 1,2
}

${ }^{1}$ Museo de Historia Natural, Av. Arenales 1256, Aptdo. 14-0434, Lima 14, Perú

2 Plant Resources Center, University of Texas at Austin, Austin TX 78712 EE.UU.

blanca.leon@mail.utexas.edu

\section{Resumen}

La familia Ericaceae es una de las familias características de los bosques montanos peruanos. Está representada en la flora del país por 22 géneros y 132 especies (Brako \& Zarucchi, 1993; Ulloa Ulloa et al., 2004), todas plantas perennes que van de arbustos a plantas trepadoras. En este trabajo reconocemos 51 especies y cuatro variedades como endemismos peruanos en 14 géneros. Thibaudia y Demosthenesia son los géneros con mayor número de especies endémicas. Estos taxones endémicos ocupan principalmente las regiones Mesoandina y Bosques Muy Húmedos Montanos, entre los 1000 y $3600 \mathrm{~m}$ de altitud. Once de estos taxones endémicos se encuentran representados dentro del Sistema Nacional de Áreas Naturales Protegidas por el Estado.

Palabras claves: Ericaceae, Thibaudia, Demosthenesia, Perú, endemismo, plantas endémicas.

\section{Abstract}

The Ericaceae, a characteristic family of Peru's montane forests, are represented in Peru by 22 genera and 132 species (Brako \& Zarucchi, 1993; Ulloa Ulloa et al., 2004), all of them perennial plants, from shrubs to climbing plants. Here we recognize 51 species and four varieties as Peruvian endemics in 14 genera. Thibaudia and Demosthenesia are the genera with the largest number of endemic species. Endemic Ericaceae are found in the Mesoandean and Very Humid Montane Forests regions, between 1000 and $3600 \mathrm{~m}$ elevation. Eleven of these endemic taxa have been collected to date within Peru's protected areas system.

Keywords: Ericaceae, Thibaudia, Demosthenesia, Peru, endemism, endemic plants.

\section{Agarista bracamonensis (Kunth) G. Don}

$$
\text { CR, B2ab(iii) }
$$

Publicación: Gen. hist. 3: 837. 1834.

Colección tipo: A. Bonpland 3564

Herbarios: F, P.

Nombre común: D esconocido.

Registro departamental: CA.

Regiones Ecológicas: MA; 2350- $2600 \mathrm{~m}$.

SINANPE: Sin registro.

Hemarios penuanos: CPUN (1), USM (1).

Observaciones: Esta especie se conoce sólo de una localidad en el norte del país. La colección más reciente proviene de 2002, de la misma localidad original; probablemente, su poca representación esté asociada a la escasa exploración botánica de los ambientes paramunos. Además, el hábitat de esta especie está sujeto a quemas intencionales que permiten inferir que hay una reducción de las condiciones adecuadas para la sobrevivencia de esta especie.

\section{Aganista subcordata (Dunal) Judd}

\section{CR, B2ab(iii)}

Publicación: J. Arnold Arbor. 65: 285. 1984.

Colección tipo: A. Mathews s.n.

Hembarios: E, G, K, L, S.

Nombre común: D esconocido.

Registro departamental: AM.

Regiones Ecológicas: MA, PAR; $2700 \mathrm{~m}$.

SINAN PE: Sin registro.

Herbarios peruanos: Ninguno.
Observaciones: Arbusto conocido solamente de la región de Chachapoyas. La última colección de esta especie fue realizada en los años 1930. En forma similar a otras plantas de los matorrales y jalca, las poblaciones de esta especie están sujetas a los incendios intencionales y otras actividades humanas que deterioran el hábitat de estas.

\section{Bejaria infundibula Clemants

$$
\text { EN, Blab(iii) }
$$

Publicación: Brittonia 43(3): 173. 1991.

Colección tipo: S.F. Clemants \& R. Fernández 2007

Herbarios: AAU, CAS, MO, NY, US; USM!.

Nombre común: Mupa sacha

Registro departamental: AM, CA.

Regiones Ecológicas: MA, BMHM; 1520-3200 m. SINANPE: PNC

Herbarios peruanos: CPUN (2), HUT (isotipo+1), USM (isotipo+1).

Observaciones: Arbusto conocido del norte del Perú, en un área aproximada de menos de $1000 \mathrm{~km}^{2}$. Ha sido recolectada varias veces en la provincia de Chachapoyas y en áreas vecinas; sólo se conoce de un registro en Cutervo. Las amenazas más importantes a las poblaciones provienen especialmente de la tala e incendios. La mayoría de las localidades conocidas se hallan fuera de áreas protegidas.

\footnotetext{
Nota del Editor: En la versión on line de este artículo han sido omitidos los mapas del Perú que ilustraban el Registro departamental. Para ubicar las abreviaturas de los departamentos vea al final del artículo.
} 


\section{Cavendishia nobilis Lindl. var. nobilis}

$$
\text { EN, Blab(iii) }
$$

Publicación:

Colección tipo: A. Mathews 2078

Hemarios: $\mathrm{K}$.

Nombre común: D esconocido.

Registro departamental: CU, HU, JU, PA, PU.

Regiones Ecológicas: BMHM; 1600$2560 \mathrm{~m}$.

SINAN PE: PNYC

Herbarios peruanos: MOL (1).

Observaciones: Esta planta se conoce de por lo menos cinco localidades a lo largo de la vertiente oriental de Huánuco a Puno. Luteyn (1983) mencionó que esta variedad puede hibridar con otras especies afines. No hay colecciones numerosas de esta variedad, tal vez por su presencia en ambientes secundarios, que son poco herborizados.

\section{Cavendishia punctata (Ruiz \& Pav. ex J. St.-Hil.) Sleumer}

$$
\text { VU, B la+b(iii) }
$$

Publicación: Notizbl. Bot. Gart. BerlinDahlem 12: 294. 1935.

Colección tipo: H. Ruiz \& J. Pavón s.n. Herbarios: MA.

Nombre común: Huehli de monte, huegli (JU).

Registro departamental: HU, JU, PA. Regiones Ecológicas: BMHM; 2100$2900 \mathrm{~m}$.

SINANPE: PNYC

Hemarios peruanos: USM (13).

Observaciones: Esta especie se conoce de varias colecciones del centro del Perú. Habita la zona del bosque húmedo montano, la cual está sujeta a fuerte presión por actividad humana, especialmente a lo largo de caminos carrozables. El área de presencia es de aproximadamente de $3000 \mathrm{~km}^{2}$. Con la excepción de una población en el Parque Nacional Yanachaga-Chemillén, no tiene poblaciones protegidas de la tala, quema y ocupación humana de su hábitat.

\section{Ceratostema callistum A.C. Sm.}

\section{EN, Blab(iii)}

Publicación: J. Wash. Acad. Sci. 46: 113. 1956.

Colección tipo: W. Rauh \& G. Hirsch 2186

Henbarios: US.

Nombre común: D esconocido.

Registro departamental: CA, LA, PI. Regiones Ecológicas: MA; 2050- 2500 m.

SINAN PE: Sin registro.

Hemarios peruanos: HAO (1), USM (3).

Observaciones: Arbusto epífito, conocido de tres localidades con poblaciones abundantes. Crece en los fragmentos de bosque de la vertiente del Pacífico y probablemente, el área de distribución no exceda los $2000 \mathrm{~km}^{2}$. Las amenazas a las poblaciones provienen de la modificación de su hábitat, pues los bosques incluyen importantes especies maderables como Pobaraus

\section{Ceratostema ferreyrae Luteyn}

\section{CR, Blab(iii)}

Publicación: J. Arnold Arbor. 67(4): 487, f. 1.1986.

Colección tipo: R. Ferreyra 18279

Hemarios: NY; USM!.

Nombre común: D esconocido.

Registro departamental: SM.

Regiones Ecológicas: BMHP; 1100$1200 \mathrm{~m}$.

SINAN PE: Sin registro.

Herbarios peruanos: USM (isotipo).

Observaciones: Arbusto conocido solamente de una localidad. No ha vuelto a ser recolectada desde los inicios de la década de 1970. O cupa una zona ecológica afectada por la deforestación y la ampliación agrícola. Probablemente pueda hallársele en el reciente creado Parque Nacional Cordillera Azul.

\section{Ceratostema rauhii Luteyn}

\section{CR, Blab(iii, iv)}

Publicación: Bull. Torrey Bot. Club 119(3): 314-315, f. 1. 1992.

Colección tipo: W. Rauh 68468

Henbarios: HEID, NY.

Nombre común: D esconocido.

Registro departamental: CA.

Regiones Ecológicas: MA; $2200 \mathrm{~m}$.

SINAN PE: Sin registro.

Herbarios peruanos: Ninguno.

Observaciones: Planta epífita conocida solamente de una localidad. Este lugar, ubicado en la vertiente Pacífica, no pertence políticamente a Lambayeque como se indica en la descripción. Al igual que otros fragmentos de bosque de la vertiente Pacífica, esta localidad está amenazada por la deforestación debido a la tala por madera y expansión agrícola. A pesar de los esfuerzos por la herborización y el estudio de estos bosques no hay colecciones adicionales.

\section{Demosthenesia amiconum (Sleumer) Sleumer}

$$
\text { CR, B 1ab(iii) }
$$

Publicación: Bot. Jahrb. Syst. 71: 404. 1941.

Colección tipo: E.P. Killip \& A.C. Smith 22298

Herbarios: NY, US.

Nombre común: D esconocido.

Registro departamental: AY.

Regiones Ecológicas: BMHM; 2500 m.

SINANPE: Sin registro.

Herbarios peruanos: Ninguno.

Observaciones: Esta especie se conoce solamente de una localidad, la cual fue erróneamente ubicada por Macbride (1959) en Junín. No ha vuelto a ser recolectada desde finales de la década de 1920. Esta localidad se ubica en unazona ecológicaamenazada por deforestación. 


\section{Demosthenesia buxifolia (Field \& Gardner) A.C. Sm.}

$$
\text { EN, Blab(iii) }
$$

Publicación: Bull. Torrey Bot. Club 63(6): 310. 1936.

Colección tipo: A. Mathews 1176

Herbarios: $\mathrm{K}$.

Nombre común: D esconocido.

Registro departamental: AY, JU.

Regiones Ecológicas: BMHM; $3000 \mathrm{~m}$.

SINANPE: Sin registro.

Hembarios peruanos: USM (3).

Observaciones: Este arbusto pequeño se conoce de la parte alta de la cuenca del Chanchamayo y de la Cordillera de Vilcabamba. Ha sido recolectada al menos en tres ocasiones en el siglo XIX, pero aparentemente sólo unas pocas veces más en el siglo XX. No fue incluida como endémica por Brako \& Zarucchi (1993). Las localidades de laque se conoce están muy impactadas por la agricultura y otras actividades humanas.

\section{Demosthenesia cordifolia Luteyn}

\section{CR, B 1ab(iii)}

Publicación: Brittonia 30(4): 432, f. 911, 15. 1978.

Colección tipo: T.R. Dudley 11196

Hembarios: NA; USM?

Nombre común: D esconocido.

Registro departamental: CU.

Regiones Ecológicas: BPM; 2900— 3410 m. SINAN PE: PNO

Herbarios penuanos: USM (isotipo+3).

Observaciones: Arbusto o liana conocido solamente de una localidad que hoy forma parte de un área protegida. No ha vuelto a ser recolectada desde fines de la década de 1960

\section{Demosthenesia dudleyi D.R. Simpson}

$$
\text { CR, Blab(iii) }
$$

Publicación: Fieldiana, Bot. 36: 4. 1972. Colección tipo: T.R. Dudley 11199

Herbarios: F, NA; USM!

Nombre común: D esconocido.

Registro departamental: CU.

Regiones Ecológicas: BPM; 3400 m.

SINAN PE: PNO

Herbarios peruanos: USM (isotipo+1).

Observaciones: Arbusto conocido solamente de una localidad; no ha vuelto a ser recolectada desde fines de la década de 1960. La localidad original se halla en la vertiente occidental del valle del Apurímac y esta área no ha sido herborizada recientemente. Los fragmentos de bosque de toda la vertiente andino-amazónica están sujetos a la destrucción por actividades agrícolas.

\section{Demosthenesia oppositifolia Luteyn}

\section{EN, B1ab(iii)}

Publicación: Brittonia 30(4): 434-435. 1978. Colección tipo: T.R. Dudley 11068

Herbarios: F, NA; USM!

Nombre común: D esconocido.

Registro departamental: CU.

Regiones Ecológicas: $\mathrm{BPM}, \mathrm{BMHM}$; 3100-3300 m.

SINANPE: PNO

Herbarios penuanos: USM (isotipo+2).
Observaciones: Arbusto conocido solamente de una localidad; no hay registro de colecciones recientes desde fines de 1960. Se sabe que se presenta en un área aproximada de $150 \mathrm{~km}^{2}$. La mayor parte de la población de esta especie está incluida en un área protegida, pero por el rango altitudinal, las que quedan fuera de esta podrían estar afectadas por la quema y deforestación.

\section{Demosthenesia vilcabambensis Luteyn} CR, Bla

Publicación: Brittonia 30(4): 435, 438439, f. 16- 18. 1978.

Colección tipo: T.R. Dudley 10840

Hemarios: NA; USM.

Nombre común: D esconocido.

Registro departamental: CU.

Regiones Ecológicas: BMHM; 2400$2665 \mathrm{~m}$.

SINAN PE: PNO

Herbarios penuanos: USM (isotipo+1).

Observaciones: Liana conocida solamente de una localidad, hoy parte de un área protegida. Esta especie parece estar restringida a bosques húmedos en laderas de quebradas pronunciadas. No ha vuelto a ser recolectada desde fines de la década de 1960.

\section{Demosthenesia weberbaueri (Sleumer) Sleumer}

\section{CR, Bla}

Publicación: Repert. Spec. Nov. Regni Veg. 41: 119. 1936.

Colección tipo: A. Weberbauer 5564

Herbarios: US.

Nombre común: D esconocido.

Registro departamental: AY.

Regiones Ecológicas: BPM; 3400 m.

SINAN PE: Sin registro.

Herbarios peruanos: USM (1).

Observaciones: Arbusto conocido de una sola localidad. El ejemplar tipo fue recolectado en 1910. Una colección adicional en la misma cuenca del A purímac fue obtenida en 1978. Probablemente se halle en ambientes ecotonales de bosque y pajonal, al igual que otras especies en el género. Las poblaciones de esta especie se hallan en la vertiente occidental del río A purímac frente al Parque Nacional Otishi, pero no reciben protección oficial.

\section{Diogenesia caudata (Sleumer) Sleumer}

\section{CR, Bla}

Publicación: Bot. Jahrb. Syst. 71: 396. 1941.

Colección tipo: A. Raimondi 9873

Henbarios: B, US; USM!.

Nombre común: Desconocido.

Registro departamental: AY.

Regiones Ecológicas: $\mathrm{BPM}, \mathrm{BMHM}$; altitud desconocida.

SINAN PE: Sin registro.

Herbarios peruanos: USM (isotipo).

Observaciones: Arbusto conocido solamente de una localidad. Poco se sabe del hábitat y rango altitudinal de esta especie, pero probablemente se ubica en las zonas montanas húmedas de la cuenca del río Apurímac. No ha vuelto a ser recolectada desde fines de los 1860. La localidad original se ubica al suroeste del Parque Nacional Otishi. 
17. Diogenesia laxa (A.C. Sm.) Sleumer

$$
\text { CR, Bla }
$$

Publicación: Bot. Jahrb. Syst. 71: 396. 1941.

Colección tipo: A. Weberbauer 7532

Herbarios: BM.

Nombre común: D esconocido.

Registro departamental: AY.

Regiones Ecológicas: PSH, BPM; 3200

m.

SINAN PE: Sin registro.

Herbarios peruanos: Ninguno.

Observaciones: Arbusto conocido solamente de una localidad, ubicada en las vertientes occidentales del valle del Apurímac. No ha vuelto a ser recolectada desde 1926. El área de la localidad original ha sido poco herborizada, en tanto que las amenazas al hábitat están asociados a la quema de pajonales y fragmentos del bosque montano.

\section{Diogenesia vargasiana Sleumer}

\section{CR, Blab(iii)}

Publicación: Notes Roy. Bot. Gard. Edinburgh 36: 257. 1978.

Colección tipo: C. Vargas C. 3025

Henbarios: US.

Nombre común: D esconocido.

Registro departamental: CU.

Regiones Ecológicas: BMHM; 2800$3000 \mathrm{~m}$.

SINANPE: PNM

Herbarios peruanos: USM (9).

Observaciones: Esta especie arbustiva se conoce del sur del país, de la cuenca alta del Madre de Dios. Ha sido recolectada en varias ocasiones más en la misma localidad. No fue incluida como endémica en Brako \& Zarucchi (1993). Si bien se conoce de una población en el Parque Nacional Manu, incendios ocasionales a esa altitud y el impacto de la carretera de acceso a la parte baja de la cuenca pueden considerarse las amenazas más importantes.

\section{Disterigma ulei Sleumer}

\section{DD}

Publicación: Bot. Jahrb. Syst. 71: 397. 1941.

Colección tipo: E.H.G. Ule 6448

Herbanios: B.

Nombre común: D esconocido.

Registro departamental: SM.

Regiones Ecológicas: BMHP; 1200 m.

SINANPE: ACRCE

Herbarios peruanos: Ninguno.

Observaciones: Arbusto conocido solamente de una localidad. No ha vuelto a ser recolectada desde inicios del siglo XX. La localidad original está hoy ubicada en el Area de Conservación Regional Cerro La Escalera. Probablemente esté representada en el Parque Nacional Cordillera Azul, al sur de la localidad original. Toda la región es un mosaico de tipos diferentes de rocas que condicionan drenaje y sostienen comunidades de plantas, pero no se conoce en cuál de estos tipos habita esta especie.

\section{Disterigma weberbaueri $\mathrm{H}$ oenold}

$$
\text { CR, B 1ab(iii) }
$$

Publicación: Bot. Jahrb. Syst. 42: 334. 1909.

Colección tipo: A. Weberbauer 2079

Herbarios: B; MOL!.

Nombre común: Desconocido.

Registro departamental: JU.

Regiones Ecológicas: MA; $3000 \mathrm{~m}$.

SINAN PE: Sin registro.

Herbarios peruanos: MOL (isotipo).

Observaciones: Arbusto descrito de una planta recolectada en la cuenca del Palca y que no ha vuelto a ser recolectada desde inicios del siglo XX, si bien herborizaciones continuas se han efectuado cerca de esa localidad, pero limitadas a los márgenes de la Carretera Central. Los fragmentos de bosque y matorrales en esa parte del país se hallan fuertemente impactados por la actividad humana.

\section{Orthaea breviflora A.C. Sm.}

\section{VU, Bla}

Publicación: Contr. U.S. Natl. Herb. 28: 513. 1932.

Colección tipo: F.W. Pennell 13972

Hemarios: $\mathrm{F}, \mathrm{PH}$.

Nombre común: D esconocido.

Registro departamental: CU.

Regiones Ecológicas: BMHM; 2200$3500 \mathrm{~m}$.

SINANPE: PNM

Herbarios peruanos: USM (6).

Observaciones: Arbusto descrito de una planta recolectada en 1925, de la cuenca alta del Alto Madre de Dios. Esta localidad está ubicada en la parte alta y occidental del Parque Nacional Manu. Al parecer, no se conocen registros adicionales.

\section{Pellegrinia coccinea (H oerold) Sleumer}

$$
\text { EN, Blab(iii) }
$$

Publicación: Notizbl. Bot. Gart. BerlinDahlem 12: 288. 1935.

Colección tipo: A. Weberbauer 2505

Herbarios: B.

Nombre común: D esconocido.

Registro departamental: HU, JU.

Regiones Ecológicas: MA; 3400 m.

SINAN PE: Sin registro.

Herbarios peruanos: Ninguno.

Observaciones: Esta especie arbustiva se conoce de cuatro localidades en el centro del país. No ha vuelto a ser recolectada desde la década de 1920, debido tal vez a lo poco herborizado de las localidades originales. El área de presencia se calcula en 250 $\mathrm{km}^{2}$. Probablemente habite los matorrales y áreas ecotonales con el bosque montano. Este tipo de vegetación está afectado en las laderas andinas orientales por las actividades agrícolas.

\section{Pellegrinia grandiflora (Ruiz \& Pav.) Sleumer}

$$
\text { EN, B lab(iii) }
$$

Publicación: Notizbl. Bot. Gart. BerlinDahlem 12: 288. 1935.

Colección tipo: H. Ruiz \& J. Pavón s.n.

Herbarios: G, L.

Nombre común: Uchu uchu.

Registro departamental: HU, JU.

Regiones Ecológicas: MA, PSH; 3400 m.

SINANPE: Sin registro.

Henbarios peruanos: Ninguno. 
Observaciones: Esta especie se conoce de cuatro localidades en el centro del país. Las colecciones más recientes datan de la década de 1920. Habita probablemente matorrales, en dos zonas ecológicas propensas a modificación de los hábitats por actividad agrícola.

\section{Pellegrinia harmsiana (H oerold) Sleumer}

NE

Publicación: Notizbl. Bot. Gart. BerlinDahlem 12: 288. 1935.

Colección tipo: A. Weberbauer 3374

Herbanios: $\mathrm{B}$.

Nombre común: D esconocido.

Registro departamental: HU.

Regiones Ecológicas: PSH, BPM; 3300$3500 \mathrm{~m}$.

SINANPE: Sin registro.

Herbarios peruanos: Ninguno.

Observaciones: Esta especie se conoce solamente de una localidad, ubicada en una zona que no ha vuelto a ser herborizada desde inicios del siglo XX. No se cuenta con información sobre el hábitat de esta especie.

\section{Pellegrinia hirsuta (Ruiz \& Pav.) Sleumer}

\section{NE}

Publicación: Notizbl. Bot. Gart. BerlinDahlem 12: 288. 1935.

Colección tipo: H. Ruiz \& J. Pavón s.n.

Herbarios: MA.

Nombre común: D esconocido.

Registro departamental: HU.

Regiones Ecológicas: BMHM; altitud desconocida.

SINAN PE: Sin registro.

Herbarios peruanos: Ninguno.

Observaciones: Esta especie arbustiva se conoce solamente de una localidad. Fue recolectada en el siglo XVIII, en una zona escasamente herborizada en el siglo XX. No se cuenta con información sobre el hábitat de esta especie ni de los detalles del tamaño de sus poblaciones.

26. Psammisia globosa A.C. Sm.

$$
\text { CR, Blab(iii) }
$$

Publicación: Contr. U.S. Natl. Herb. 28: 388. 1932.

Colección tipo: A. Mathews 2077

Herbarios: $\mathrm{K}$.

Nombre común: D esconocido.

Registro departamental: JU.

Regiones Ecológicas: BMHP; $1000 \mathrm{~m}$.

SINAN PE: Sin registro.

Herbarios peruanos: Ninguno.

Observaciones: Esta especie arbustiva solamente se conoce del valle del Chanchamayo. Aparentemente no ha vuelto a ser recolectada desde la década de 1920 . No se conoce sobre el hábitat de esta especie; sin embargo, las localidades están afectadas por deforestación.
27. Satyria polyantha A.C. Sm.

$$
\text { CR, B lab(iii) }
$$

Publicación: Bull. Torrey Bot. Club 60: 119. 1933.

Colección tipo: W. Lechler 2068

Hemarios: B, K, P.

Nombre común: Huilunto.

Registro departamental: PU.

Regiones Ecológicas: BMHM; 2000$3000 \mathrm{~m}$.

SINAN PE: Sin registro.

Herbarios peruanos: USM (2).

Observaciones: Esta especie arbustiva fue descrita de un ejemplar recolectado en la cuenca del San Gabán. Otras dos colecciones que representan a esta especie fueron realizadas por Raimondi en 1864 y en la misma localidad; no existen, sin embargo, colecciones recientes. No hay datos adicionales del hábitat, pero probablemente habite fragmentos de bosque.

\section{Satyria vargasii A.C. Sm.}

\section{CR, Blab(iii)}

Publicación: J. Wash. Acad. Sci. 43: 212. 1953. Colección tipo: C. Vargas C. 6493

Henbarios: US.

Nombre común: D esconocido.

Registro departamental: CU.

Regiones Ecológicas: BMHP; $1360 \mathrm{~m}$.

SINAN PE: Sin registro.

Hemarios peruanos: CUZ (1).

Observaciones: Arbusto conocido solamente de una localidad. No hay colecciones adicionales desde fines de la década de 1960, probablemente por la poca herborización en lazona montana oriental.

\section{Sphyrospermum buesii A.C. Sm.}

\section{EN, Blab(iii)}

Publicación: J. Wash. Acad. Sci. 43: 208. 1953. Colección tipo: C. Bües 2165

Henbarios: US.

Nombre común: D esconocido.

Registro departamental: CU, SM.

Regiones Ecológicas: BMHM; 1750$2750 \mathrm{~m}$.

SINAN PE: Sin registro.

Herbarios penuanos: AMAZ?.

Observaciones: Este arbusto epífito se conoce solamente de dos localidades, separadas entre ellas por más de $800 \mathrm{~km}$. Lafranja de bosque montano entre estas localidades ha sido poco herborizada y esto tal vez contribuya a la escasez de colecciones. Amenazas al bosque montano incluyen deforestación e incendios forestales por la actividad agnícola que contribuyen a la fragmentación del hábitat de esta especie.

30. Sphyrospermum weberbaueri (Hoer.) A.C. Sm.

\section{NE}

Publicación: Brittonia 1(4): 212-213. 1933.

Colección tipo: A. Weberbauer 2122

Herbarios: $B$.

Nombre común: Desconocido.

Registro departamental: HU, JU.

Regiones Ecológicas: BMHM; 2000$2600 \mathrm{~m}$.

SINANPE: Sin registro.

Herbarios peruanos: USM (isotipo)? 
Observaciones: Esta especie arbustiva se conoce del centro del país de dos localidades, en las cuencas del Tulumayo y Pozuzo; pero, no hay colecciones recientes desde inicios de la década de 1920.

\section{Thibaudia angustifolia H ook.}

$$
\text { CR, Blab(iii) }
$$

Publicación: Icon. Pl. 2: t. 110. 1837.

Colección tipo: A. Mathews 1443

Herbarios: K.

Nombre común: D esconocido.

Registro departamental: AM.

Regiones Ecológicas: Sin datos; altitud desconocida.

SINANPE: Sin registro.

Herbarios peruanos: Ninguno.

Observaciones: Especie arbustiva que no fue señalada por Brako \& Zarucchi (1993) como endémica, pero que se conoce de unas pocas localidades entre las cuencas del Marañón y Utcubamba. Ha sido recolectada en Cerros Calla Calla, una localidad que alberga varios otros endemismos. Amenazas a sus poblaciones de debe a que los bosques húmedos están siendo reducidos y fragmentados por la activida agrícola.

\section{Thibaudia apophysata H oenold}

\section{CR, Blab(iii)}

Publicación: Bot. Jahrb. Syst. 42: 314. 1909.

Colección tipo: A. Weberbauer 2071

Herbarios: B; MOL!.

Nombre común: Desconocido.

Registro departamental: JU.

Regiones Ecológicas: MA; $2600-3000$ $\mathrm{m}$.

SINANPE: Sin registro.

Herbarios penuanos: MOL (isotipo).

Observaciones: Esta especie arbustiva es conocida solamente de una localidad, ubicada en el centro del país. No ha vuelto a ser recolectada desde inicios del siglo XX. Toda la zona centro del país, en especial la vertiente oriental amazónica donde se recolectó esta especie, necesita mayor herborización. D esde la visita botánica de Weberbauer, en 1903, la zona ha venido siendo modificada por actividades agrícolas.

\section{Thibaudia biflora (Poepp. \& Endl.) Hoemold}

$$
\text { EN, Blab(iii) }
$$

Publicación: Bot. Jahrb. Syst. 42: 274. 1909.

Colección tipo: E.F. Poeppig 1350

Herbarios: W.

Nombre común: Desconocido.

Registro departamental: HU, PA, SM.

Regiones Ecológicas: BMHM, BMHP; altitud desconocida.

SINANPE: Sin registro.

Herbarios peruanos: Ninguno.

Observaciones: Esta especie no fue incluida por Brako \& Zarucchi (1993) como endémica, pero se conoce solamente de tres localidades en el nor-centro del país. La recolección más reciente que se conoce fue hecha a fines de la década de 1920. Esta especie está presente en la franja boscosa de la vertiente oriental a lo largo de más de $300 \mathrm{~km}$ entre las localidades más distantes. Amenazas a las poblaciones de esta especie probablemente estén asociadas a deforestación.

\section{Thibaudia cardiophylla Sleumer}

\section{CR, B 1ab(iii)}

Publicación: Notizbl. Bot. Gart. BerlinDahlem 12: 135. 1934.

Colección tipo: A. Weberbauer 3358

Herbarios: B.

Nombre común: D esconocido.

Registro departamental: $\mathrm{HU}$.

Regiones Ecológicas: BPM; 3200-3300

m.

SINAN PE: Sin registro.

Herbarios peruanos: Ninguno.

Observaciones: Arbusto conocido solamente de una localidad. Weberbauer (1945) describió este lugar, el cual es típico de las partes más altas de la vertiente oriental andina. No hay recolecciones recientes. En general la zona ha sido poco herborizada. Amenazas a sus poblaciones están asociadas a la expansión agrícola.

\section{Thibaudia croatii Luteyn}

\section{CR, Bla+2a}

Publicación: Brittonia 29(2): 180. 1977.

Colección tipo: T. Croat 21089

Herbarios: MO, NY.

Nombre común: D esconocido.

Registro departamental: $\mathrm{HU}$.

Regiones Ecológicas: BMHM, BMHP; 1550- $1600 \mathrm{~m}$.

SINANPE: Sin registro.

Hembarios penuanos: USM (2).

Observaciones: Esta especie hemiepífita se conoce solamente del valle del río Huallaga. Probablemente se encuentre representada en el Parque Nacional de Tingo María. No ha vuelto a ser recolectada desde inicios de 1980, tal vez por la inaccesibilidad durante las últimas décadas para la herborización. Amenazas a sus poblaciones podrían estar asociadas a deforestación, aunque por su hábitat tal vez no estén afectadas.

\section{Thibaudia diphylla Dunal}

$$
\text { CR, B 1ab(iii) }
$$

Publicación: Prodr. 7: 562. 1839.

Colección tipo: J. D ombey s.n.

Herbarios: P.

Nombre común: D esconocido.

Registro departamental: JU.

Regiones Ecológicas: MA; altitud desconocida.

SINAN PE: Sin registro.

Henbarios peruanos: Ninguno.

Observaciones: Esta especie arbustiva no fue incluida por Brako \& Zarucchi (1993) como endémica, pero se conoce solamente del centro del Perú. La recolección botánica más reciente fue efectuada a fines de la década de 1920. Los lugares de recolecta se hallan en la cuenca alta del Perené. Esta parte de la cuenca está vinculada a actividades agrícolas, que tal vez promuevan la deforestación y modificación del hábitat de esta especie. 


\section{Thibaudia dudleyi Luteyn var. dudleyi}

$$
\text { CR, Blab(iii) }
$$

Publicación:

Colección tipo: T.R. Dudley 10750

Herbarios: NA; USM.

Nombre común: Desconocido.

Registro departamental: CU.

Regiones Ecológicas: BMHM; 2300$2600 \mathrm{~m}$.

SINAN PE: PNO

Herbarios peruanos: USM (isotipo citado+1).

Observaciones: Planta trepadora, conocida de una localidad, hoy incluida en un área protegida. Todos los ejemplares conocidos provienen del año 1968 y no hay recolectas adicionales. No se conoce de amenazas a esta especie en las localidades conocidas, pero la deforestación es causa de preocupación fuera de zonas protegidas.

\section{Thibaudia dudleyi Luteyn var. pseudostellata Luteyn CR, Blab(iii)}

Publicación: Brittonia 30(4): 430, 432, f. 8, 12-14. 1978.

Colección tipo: M.T. Madison 10364-70

Herbarios: F, NA.

Nombre común: D esconocido.

Registro departamental: AY.

Regiones Ecológicas: PSH, BPM; 3220$3629 \mathrm{~m}$.

SINAN PE: Sin registro.

Herbarios peruanos: USM (1).

Observaciones: Este arbusto se conoce solamente de una localidad. Al igual que la variedad típica, proviene de la cuenca del Apurímac, pero del flanco occidental que no recibe protección oficial. No hay colecciones recientes desde 1968, probablemente por la escasa herborización en la zona, la cual ha sido reconocida por tener vacío de información botánica (Rodríguez \& Young, 2000). Amenazas potenciales a las poblaciones de esta especie están asociadas a la actividad agrícola.

\section{Thibaudia engleniana H oenold}

\section{CR, B lab(iii)}

Publicación: Bot. Jahrb. Syst. 42: 312. 1909.

Colección tipo: A. Weberbauer 2055

Herbarios: B.

Nombre común: D esconocido.

Registro departamental: JU.

Regiones Ecológicas: BMHM; 3000$3100 \mathrm{~m}$.

SINAN PE: Sin registro.

Herbarios peruanos: Ninguno.

Observaciones: Este arbusto trepador se conoce solamente de una localidad. Ha sido recolectada en la misma localidad luego de un lapso de casi 80 años. En ambas colecciones, las plantas estaban en flor en el mes de enero. La falta de colecciones de herbario adicionales pueda deberse a que su floración ocurre en un lapso corto de tiempo. A esto se agrega el hecho que el hábitat de esta especie poco se herboriza. La localidad original está siendo afectada por la ampliación de la frontera agrícola y desarrollo de vías de comunicación.

\section{Thibaudia harmsiana Hoenold}

CR, B 1ab(iii)

Publicación: Bot. Jahrb. Syst. 42: 314. 1909.

Colección tipo: A. Weberbauer 3542

Henbarios: B, NY.

Nombre común: D esconocido.

Registro departamental: $\mathrm{HU}$.

Regiones Ecológicas: BMHM; 1550$2500 \mathrm{~m}$.

SINAN PE: Sin registro.

Herbarios peruanos: Ninguno.

Observaciones: Esta especie se conoce solamente de una localidad. No ha vuelto a ser recolectada desde 1939. La localidad original en Huánuco está en una de las áreas menos herborizada del centro del país. No se cuenta con información sobre el hábitat, pero por el rango altitudinal se infiere que actividades relacionadas con la agricultura y construcción vial pueden ser amenazas para las poblaciones.

\section{Thibaudia hermerae A.C. Sm.}

\section{DD}

Publicación: Contr. U.S. Natl. Herb. 28: 435-436. 1932.

Colección tipo: F. Herrera 1384

Herbarios: US.

Nombre común: D esconocido.

Registro departamental: CU.

Regiones Ecológicas: BMHM; 2000$2800 \mathrm{~m}$.

SINAN PE: Sin registro.

Herbarios peruanos: Ninguno.

Observaciones: Esta especie se conoce solamente de una localidad. Fue recolectada a fines de la década de 1920. No se conoce sobre su hábitat.

\section{Thibaudia mellifera Ruiz \& Pav. ex St. H ill.}

$$
\text { EN, B1ab(iii) }
$$

Publicación: Expos. Fam. Not. 1: 363. 1805.

Colección tipo: H. Ruiz \& J. Pavón s.n.

Herbarios: G, MO.

Nombre común: D esconocido.

Registro departamental: $\mathrm{HU}, \mathrm{JU}, \mathrm{PU}$.

Regiones Ecológicas: BMHM; 1580$2600 \mathrm{~m}$

SINAN PE: Sin registro.

Herbarios peruanos: USM (6).

Observaciones: Esta especie no fue incluida por Brako \& Zarucchi (1993) como endémica, pero se conoce principalmente del centro del país, de dos cuencas importantes, la del Huallaga y la del Perené. O tra localidad disyunta proviene de la cuenca del San Gabán. Probablementelafalta de interés en el grupo y la escasa herborización en el rango altitudinal de esta especie contribuyan a la poca representación en colecciones científicas. Sin lugar a dudas, el área de presencia de esta especie está sujeta a la presión agrícola. 


\section{Thibaudia monicandii Dunal var. phyllineaefolia (Dunal) J.F. Macbr.}

\section{NE}

Publicación: Field Mus. Nat. Hist., Bot. Ser. 13(5/ 1): 109. 1959.

Colección tipo: A. Mathews 3041 pp

Herbarios: K.

Nombre común: D esconocido.

Registro departamental: AM.

Regiones Ecológicas: Sin datos; altitud desconocida.

SINANPE: Sin registro.

Herbarios peruanos: Ninguno.

Observaciones: Se conoce solamente de una localidad en el norte del país. Poco se sabe del hábitat de esta especie ni de la localidad; se presume que habita fragmentos de bosque montano. No ha vuelto a ser recolectada desde 1835 .

\section{Thibaudia obovata A.C.Sm.}

\section{CR, Blab(iii)}

Publicación: Contr. U.S. Natl. Herb. 28(2): 431. 1932.

Colección tipo: A. Weberbauer 7154

Herbarios: B, F.

Nombre común: D esconocido.

Registro departamental: AM.

Regiones Ecológicas: BMHM; 2900$3000 \mathrm{~m}$.

SINANPE: Sin registro.

Herbarios peruanos: Ninguno.

Observaciones: Arbusto conocido solamente de una localidad en el norte del país. No ha vuelto a ser recolectada desde 1915. Weberbauer (1945) describe el ambiente de esa parte del país y por ello se sabe que habita bosques ecotonales con pajonales. Al no estar representada en un área protegida, las amenazas a sus poblaciones son similares para otras especies de los bosques montanos orientales, es decir provenientes del pastoreo y la quema asociada.

45. Thibaudia ovalifolia A.C. Sm.

\section{DD}

Publicación: Contr. U.S. Natl. Herb. 28(2): 434-435. 1932.

Colección tipo: A. Mathews 883

Herbarios: $\mathrm{K}$.

Nombre común: D esconocido.

Registro departamental: JU.

Regiones Ecológicas: Sin datos; altitud desconocida.

SINANPE: Sin registro.

Herbarios peruanos: Ninguno.

Observaciones: Arbusto descrito de una planta recolectada en el siglo X IX, del centro del país. Esta especie no fue incluida por Brako \& Zarucchi (1993) como endémica.
46. Thibaudia rauhii A.C. Sm.

$$
\text { CR, B 1ab(iii) }
$$

Publicación: J. Wash. Acad. Sci. 46(4): 114. 1956.

Colección tipo: W. Rauh P1319

Henbarios: US; USM!

Nombre común: D esconocido.

Registro departamental: $\mathrm{CU}$.

Regiones Ecológicas: BMHM; 2800$3120 \mathrm{~m}$.

SINAN PE: Sin registro.

Hembarios penuanos: USM (isotipo+1).

Observaciones: Esta especie se conoce sólo de dos colecciones del Departamento de Cusco. El valle del Marcapata, de donde se conoce esta especie, está sujeto a una deforestación intensiva; sin embargo, ninguna población está registrada de un área protegida. Las colecciones que representan esta especie se ubican en un área menor alos $10 \mathrm{~km}^{2}$. No se tieneinformación sobre los requerimientos de hábitat, pero las amenazas a las poblaciones provienen de los mismos procesos que afectan todo el bosque montano.

\section{Thibaudia spathulata A.C. Sm.}

$$
\text { CR, B lab(iii) }
$$

Publicación: Contr. U.S. Natl. Herb. 28(2): 434. 1932.

Colección tipo: A. Weberbauer 7530

Herbarios: $F$.

Nombre común: D esconocido.

Registro departamental: AY.

Regiones Ecológicas: $\mathrm{BPM}, \mathrm{BMHM}$; $3300 \mathrm{~m}$.

SINANPE: Sin registro.

Herbarios peruanos: Ninguno.

Observaciones: Esta especie se conoce de una localidad, en la cuenca alta del río A purímac. Sólo la colección de 1926 representa a esta especie, que se sabe habita una de las zonas menos conocida botánicamente y fuertemente impactada por la ampliación agrícola.

\section{Thibaudia tomentosa H oenold}

$$
\text { CR, B lab(iii) }
$$

Publicación: Bot. Jahrb. Syst. 42: 312. 1909.

Colección tipo: A. Weberbauer 4374

Hemarios: B, F; MOL!.

Nombre común: Desconocido.

Registro departamental: AM, CA.

Regiones Ecológicas: MA; 2200- 2400 $\mathrm{m}$.

SINAN PE: Sin registro.

Herbarios penuanos: CPUN (2), HUT (1), MOL (isotipo), USM (1)?.

Observaciones: Se conoce solamente del norte del país. La localidad original fue descrita por el mismo Weberbauer (1945) como una zona de vegetación variada y flora diversa. Esta especie ha vuelto a ser recolectada recientemente. Las localidades están ubicadas en un área reconocida como prioritaria para conservación. Amenazas potenciales a las poblaciones están asociadas a deforestación para fines agropastoriles. 
49. Thibaudia uniflora A.C. Sm.

DD

Publicación: Amer. J. Bot. 27: 543. 1940.

Colección tipo: Y. Mexia 8319

Herbarios: MO, NY, US.

Nombre común: D esconocido.

Registro departamental: HU.

Regiones Ecológicas: BMHP; $825 \mathrm{~m}$.

SINAN PE: Sin registro.

Herbarios peruanos: Ninguno.

Observaciones: Esta especie arbustiva se conoce solamente de una localidad, en la cuenca del Huallaga. El ejemplar tipo fue recolectado en 1936 y aparentemente no hay registros adicionales.

\section{Thibaudia urbaniana Hoemold}

\section{CR, B lab(iii)}

Publicación: Bot. Jahrb. Syst. 42: 315. 1909.

Colección tipo: A. Weberbauer 4449

Herbarios: B.

Nombre común: D esconocido.

Registro departamental: AM.

Regiones Ecológicas: MA; 2200- 2300 m.

SINANPE: Sin registro.

Hemarios peruanos: Ninguno.

Observaciones: Esta especie arbustiva se conoce de una localidad en la cuencamedia del nío Utcubamba. No hay colecciones adicionales desde 1904. Habita en unazona con vegetación húmeda fragmentada por la influencia de su localización en la sombra de lluvia. Weberbauer (1945) brevemente señala para un área vecina las características de una vegetación mesoandina. Amenazas a las poblaciones de esta especie pueden estar asociadas a la ganadería.

\section{Vaccinium didymanthum Dunal}

$$
\text { EN, B1a+2a }
$$

Publicación: Prodr. 7: 575. 1839.

Colección tipo: A. Mathews s.n.

Herbarios: G.

Nombre común: Mote sacha.

Registro departamental: AM, AY, CA, SM.

Regiones Ecológicas: $\mathrm{MA}, \mathrm{BMHM}$; 2900-3100 m.

SINAN PE: Sin registro.

Hemarios peruanos: CPUN (1), HAO (1), USM (2).

Observaciones: Se conoce de cuatro localidades. Las poblaciones del norte del país se hallan naturalmente fragmentadas en la cuenca media del río Marañón. No se ha confirmado la distribución en Ayacucho. El área de presencia de esta especie en el norte del país no supera los $100 \mathrm{~km}^{2}$. La deforestación constituye la amenaza principal a las poblaciones de esta especie.

\section{Vaccinium elvirae Luteyn}

\section{CR, B lab(iii)}

Publicación: Opera Bot. 92: 129. 1987.

Colección tipo: J. Luteyn \& E. Cotton 11381

Hembarios: AAU, CAS, F, G, MO, NY, US;

HUT!, USM!.

Nombre común: D esconocido.

Registro departamental: AM, CA.

Regiones Ecológicas: BMHM; 2870$3000 \mathrm{~m}$.

SINAN PE: Sin registro.
Herbarios penuanos: CPUN (2), HAO (1), HUT (isotipo), USM (isotipo+1).

Observaciones: Esta especie se conoce de cuatro localidades. Parece ser especialista de suelos arenosos y probablemente esto contribuya al tamaño reducido de sus poblaciones. Podría estar representada en otras cordilleras aisladas vecinas.

\section{Vaccinium mathewsii Sleumer}

$$
\text { EN, Bla }
$$

Publicación: Notizbl. Bot. Gart. BerlinDahlem 13: 134. 1936.

Colección tipo: A. Mathews s.n.

Herbarios: K.

Nombre común: D esconocido.

Registro departamental: AM.

Regiones Ecológicas: BMHM; 2075$3300 \mathrm{~m}$.

SINANPE: Sin registro.

Hembarios penuanos: USM (6).

Observaciones: Esta especie se conoce de cuatro localidades. Sólo ha sido registrada en el centro y sur de Amazonas de un área de presencia aproximada de $2000 \mathrm{~km}^{2}$.

\section{Vaccinium pseudocaracasanum Sleumer}

$$
\text { EN, Blab(iii) }
$$

Publicación: Notizbl. Bot. Gart. BerlinDahlem 12: 139. 1934.

Colección tipo: A. Weberbauer 4347

Hemarios: B.

Nombre común: D esconocido.

Registro departamental: AM.

Regiones Ecológicas: $\mathrm{MA}, \mathrm{BMHM}$; 2900- $3200 \mathrm{~m}$.

SINAN PE: Sin registro.

Herbarios peruanos: USM (3).

Observaciones: Esta especie fue descrita de una localidad en la cuenca media del río Utcubamba. Weberbauer (1945) brevemente describió que la vegetación es variaday laflora diversa, deduciéndose la presencia de un mosaico de bosque y pajonales húmedos. Las colecciones más recientes datan de la década de 1960 y provienen de bosques montanos húmedos. Este tipo de bosque, en el D epartamento de Amazonas, está fuertemente impactado por la deforestación.

\section{Vaccinium sphyrospermoides Sleumer}

\section{DD}

Publicación: Notizbl. Bot. Gart. BerlinD ahlem 13: 137. 1937.

Colección tipo: A. Mathews 1466

Herbarios: $\mathrm{K}$.

Nombre común: D esconocido.

Registro departamental: AM.

Regiones Ecológicas: BMHM; altitud desconocida.

SINANPE: Sin registro.

Herbarios penuanos: USM (2)?.

Observaciones: Esta especie no fue incluida por Brako \& Zarucchi (1993) como endémica, pero se conoce solamente del norte del país, en la cuenca del Marañón. 
Mapa del Perú indicando las abreviaturas de los departamentos

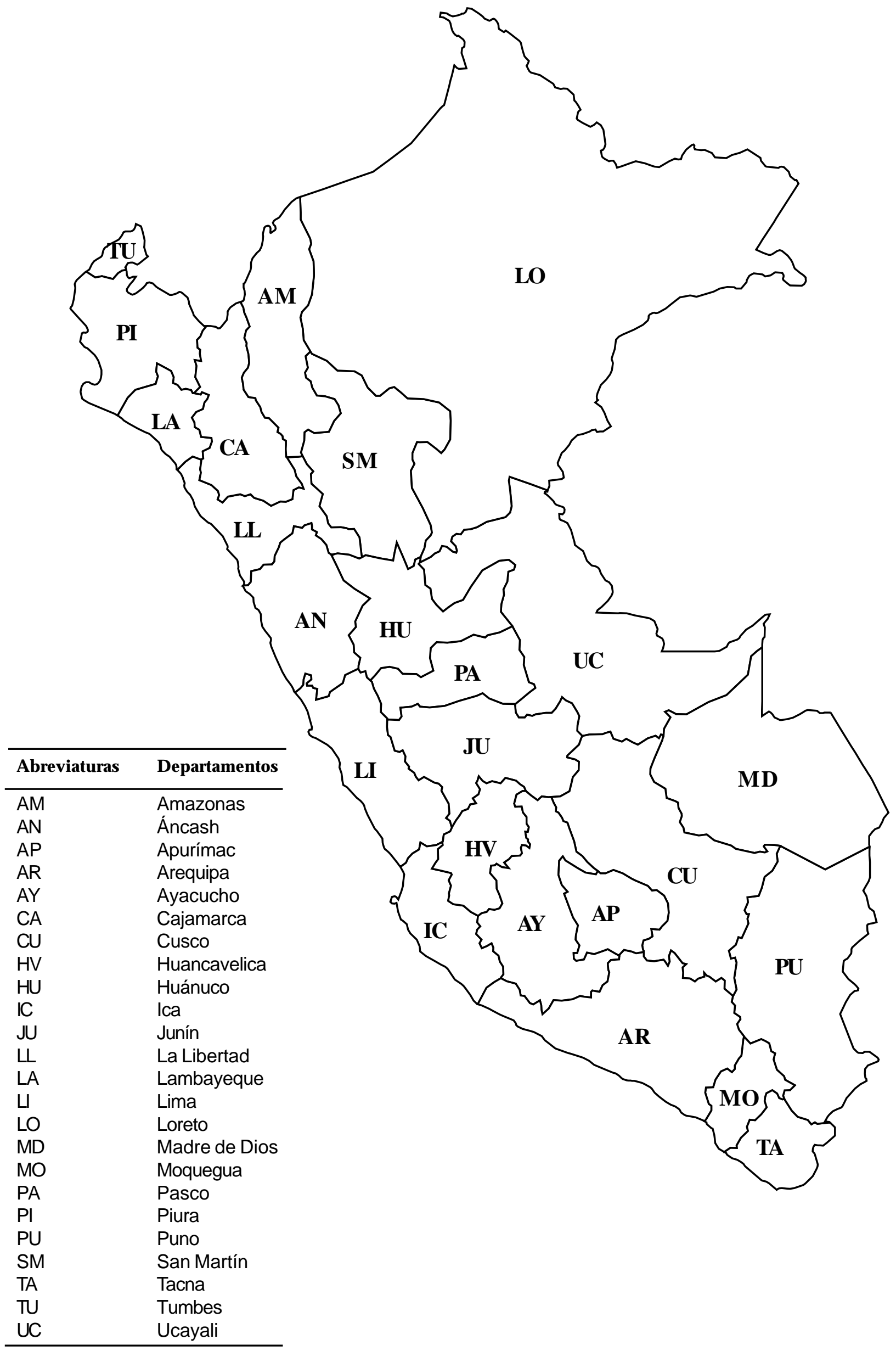

